\title{
Investigation of the modulation between EEG alpha waves and slow/fast delta waves in children in different depths of Desflurane anesthesia
}

\section{Exploration des relations de modulation entre les ondes alpha et les bandes delta rapide et delta lente de l'EEG chez l'enfant à différents niveaux d'anesthésie sous Desflurane}

\author{
B. Molaee-Ardekani ${ }^{\text {a,b,c }}$, M.B. Shamsollahi ${ }^{\text {c }}$, O. Tirel ${ }^{\text {a,b,d }}$, B. Vosoughi-Vahdat ${ }^{\text {c }}$, \\ E. Wodey ${ }^{a, b, d}$, L. Senhadji ${ }^{a, b, *}$ \\ a Inserm, U642, 35000 Rennes, France \\ b LTSI, université de Rennes 1, 35000 Rennes, France \\ ${ }^{c}$ BiSIPL, School of Electrical Engineering, Sharif University of Technology, Tehran, Iran \\ d Service d'anesthésie réanimation 2, CHU de Rennes, 35000 Rennes, France \\ Received 7 June 2009; accepted $1^{\text {st }}$ October 2009 \\ Available online 27 November 2009
}

\begin{abstract}
Objectives. - Investigation of the amplitude modulation of alpha-band EEG oscillations (i.e., grouping of alpha-band activities) by delta-band EEG activities in various depths of anesthesia (DOA).

Methods. - This modulation, which is a sort of phase dependent amplitude modulation, is studied in 10 children in various depths of Desflurane anesthesia. Two parameters are defined to quantify the modulation: strength of modulation (SOM) and phase of modulation (POM). SOM indicates to what extent delta and alpha activities are related to each other, and POM is the delta phase in which the alpha amplitude is maximal. These parameters are analyzed in different DOA for various formations of delta sub-bands.

Results. - The ability of POM and SOM were explored to characterize mechanisms contributing to delta activities and their correlation with the level of anesthesia. These parameters are influenced by DOA and frequency intervals of delta sub-bands. SOM takes higher values around certain frequency ranges of delta band. According to this, delta band comprises three main sub-bands in various unconsciousness levels. Although boundaries of these sub-bands change with DOA, they are almost in [0.1-0.5] Hz (very slow delta), [0.7-1.7] Hz (slow delta) and [2-4] Hz (fast delta) intervals. POMs relating to slow and fast delta bands increase with consciousness level. This is an indication that delta waves differently modulate alpha EEG activities (in terms of phase lag) in different DOA. In deep anesthesia, POM relating to fast delta correlates with DOA better than POM relating to slow delta does. In light anesthesia this correlation is inversed. Investigation regarding to different formations of delta sub-bands shows that POM relating to [1.8-4] Hz is a proper choice for distinguishing deep, moderate and light anesthesia.

Conclusion. - SOM allows separating mechanisms underlying delta band activities, and POM can be seen as a complementary neurophysiologicbased parameter for quantifying DOA.
\end{abstract}

(C) 2009 Elsevier Masson SAS. All rights reserved.

Keywords: Modulation; Phase coupling; EEG; Slow Delta; Fast Delta; Delta band segmentation; Desflurane Anesthesia; Depth of Anesthesia; BIS ${ }^{\mathrm{TM}}$

\section{Résumé}

Dans le présent article, la modulation des ondes Alpha par des activités EEG plus lentes est étudiée à diverses profondeurs d'anesthésie sous Desflurane. Cette modulation, qui de type modulation d'amplitude dépendante de la phase, est considérée dans le cas de dix enfants. La modulation est mesurée par le biais de deux paramètres appelés respectivement phase de la modulation et taux de la modulation (POM, SOM). Ces paramètres sont calculés pour différents regroupements de sous-bandes de la bande Delta et sont utilisés pour isoler différents mécanismes neurophysiologiques contribuant à la génération de l'activité dans la bande Delta, et pour déterminer la profondeur d'anesthésie. L'interprétation du paramètre SOM

\footnotetext{
* Corresponding author. LTSI, campus de Beaulieu, université de Rennes1, bâtiment 22, $5^{\mathrm{e}}$ étage, 35042 Rennes, France.

E-mail addresses: molaee-ardekani@ieee.org, behnam.molaee-ardekani@univ-rennes1.fr (B. Molaee-Ardekani), mbshams@ sharif.edu (M.B. Shamsollahi), olivier.tirel@chu-rennes.fr (O. Tirel), vahdat@sharif.edu (B. Vosoughi-Vahdat), eric.wodey@chu-rennes.fr (E. Wodey), lotfi.senhadji@univ-rennes1.fr (L. Senhadji).
} 
indique que la bande Delta comporte trois sous-bandes principales correspondant approximativement aux intervalles [0,1-0,5], [0,7-1,7] et [2-4] $\mathrm{Hz}$ (activité très lente, lente et rapide). Néanmoins, les limites de ces bandes sont susceptibles de varier avec la profondeur d'anesthésie. Les variations de POM en lien avec le volume du Désflurane indiquent que ce paramètre peut contribuer à l'évaluation de la profondeur d'anesthésie. En anesthésie profonde, le paramètre POM relatif à l'activité rapide est plus corrélé à la profondeur d'anesthésie que ne l'est le paramètre POM relatif à l'activité lente. Sous anesthésie légère, cette dernière situation est inversée. Les analyses statistiques comparatives menées montrent que le paramètre POM pour la bande [1,8-4] Hz permet de distinguer correctement les niveaux d'anesthésie profonde, modérée et légère.

(C) 2009 Elsevier Masson SAS. Tous droits réservés.

Mots clés : Modulation ; Couplage de phase ; EEG ; Delta rapide ; Delta lent ; Sous-bandes Delta ; Anesthésie ; Profondeur d'anesthésie ; Desflurane ; BIS ${ }^{\mathrm{TM}}$

\section{Introduction}

When an anesthetic agent is inducted and anesthesia deepens, the electroencephalogram (EEG) becomes more regular before disappearing into an isoelectric activity in very deep anesthesia. In moderate to deep anesthesia the EEG is dominated by globally coherent slow waves activities in the delta frequency range [1]. Scalp EEG as well as single neurons show that delta band may comprise different types of activities. Benoit et al., by the use of scalp EEG power spectra during non-REM sleep showed that slow and fast delta components differently correlate to alpha and beta frequency bands [2]. They considered [0.7-2] $\mathrm{Hz}$ interval as slow delta and [2-4] $\mathrm{Hz}$ interval as fast delta. Studies on neural activities by Steriade et al. [3,4], showed that slow oscillation $(<1 \mathrm{~Hz})$ has the ability to trigger and group cortical network firing, which correspond to higher frequency EEG activities from delta $([1-4] \mathrm{Hz})$ to gamma $([30-60] \mathrm{Hz})$. Steriade et al., indicated (by the means of neuronal recordings) that the hyperpolarized phase of slow oscillation is associated with a global dysfacilitation in corticothalamic networks resulting in reduced neural firing. The depolarizing phase, on the other hand, is found to be accompanied by a corticothalamic facilitation of neural firing. Fell et al., used the scalp EEG data during sleep and showed that sigma activity $([12-16] \mathrm{Hz})$ is modulated by slow EEG oscillations [5]. However, they did not find a strong modulation between slow delta waves and gamma oscillations on scalp EEG recordings as Steriade et al., had shown in their neuronal level recordings. In another study, Molle et al., found that grouping of spindle $([12-15] \mathrm{Hz})$ and fast activities in the beta band $([15-25] \mathrm{Hz})$ are coincident with slow oscillations in human slow wave sleep [6]. Interestingly, it has been also shown that infraslow oscillations $([0.02-0.2] \mathrm{Hz})$ are also strongly synchronized with faster activities, as well as with the interictal epileptic events and K-complexes [7].

All above-mentioned studies examine the EEG or neural activities during sleep or light Ketamine-Xylazine anesthesia. It remained unclear, however, whether or not the modulation of high-frequency EEG activities by slow oscillations (in the delta band) is independent of the level of unconsciousness induced by anesthetic agents. In this study, we use Desflurane agent and investigate the amplitude modulation of alpha waves by different frequency components of delta band. Two parameters are defined to characterize this modulation: phase of modulation (POM) and strength of modulation (SOM). These parameters have neurophysiologic bases, and somehow show the status of information processing in the brain. They are analyzed in 10 children in different depths of anesthesia (DOA). SOM indi- cates to what extent delta and alpha activities are related to each other in terms of amplitude modulation, and POM is the delta phase in which the alpha amplitude is maximal. Investigation into these parameters in microscopic and macroscopic scales not only has theoretical benefits but also may find some applications in-patient monitoring techniques. As an example, in this article we show that POM can be helpful for quantifying DOA. We also use SOM as a criterion to identify different types of delta band components.

This paper is organized as follows. The methodology is given in the next section. It contains description of EEG recording protocol and signal processing required for deriving POM and SOM parameters. These parameters are reported for a single patient to help readers become more familiar with POM and SOM concepts. Results are reported in section 3 and finally, discussions and suggestions for future studies form the last section of this paper.

\section{Materials and methods}

\subsection{Data recording protocol}

After approval from the human studies committee, 10 children (eight males, two females, age $105 \pm 21$ month) requiring elective surgery were recruited into the study with parental consent. None of the children was premedicated. EEG electrodes (3 M Red Dot Silver/Silver Chloride model 2269T, $3 \mathrm{M}$ Health Care, St Paul, USA) were placed adjacent to the pediatric Bispectral Index (BIS ${ }^{\mathrm{TM}}$ ) electrodes (Aspect $\mathrm{XP}^{\mathrm{TM}}$ Medical Systems, Newton, IL, USA), which are located in the frontal region of the brain $\left(\mathrm{F}_{\mathrm{p}}, \mathrm{F}_{7}\right)$. BIS ${ }^{\mathrm{TM}}$ monitor, which is a commercial device (and thus it is a black box system), integrates several disparate descriptors of the EEG into a single variable which is called $\mathrm{BIS}^{\mathrm{TM}}$ [8]. These descriptors are burst suppression ratio [9], Beta ratio $\left(\log \left[\mathrm{P}_{30-47 \mathrm{~Hz}} / \mathrm{P}_{10-20 \mathrm{~Hz}}\right]\right)$, and SynchFastSlow $\left(\log \left[\mathrm{B}_{0.5-47 \mathrm{~Hz}} / \mathrm{B}_{40-47 \mathrm{~Hz}}\right]\right)[10]$. P stands for power spectrum, and $\mathrm{B}$ stands for bispectrum. To derive these parameters, it is required that EEG is analyzed in time, frequency and bispectral [11] domains, respectively.

All EEG recordings (PowerLab ${ }^{\mathrm{TM}}$, ADInstruments, Castle Hill, NSW, Australia) were started a few minutes after the intubation when age corrected expired Desflurane concentrations [12] were stabilized at 2 MAC. For a 10-year old child, Desflurane concentration is almost $7.5 \mathrm{Vol} \%$ at $1 \mathrm{MAC}$. According to definition, $1 \mathrm{MAC}$ is the Minimum Alveolar Concentration of an anesthetic agent at 1 atmosphere pressure at which $50 \%$ 


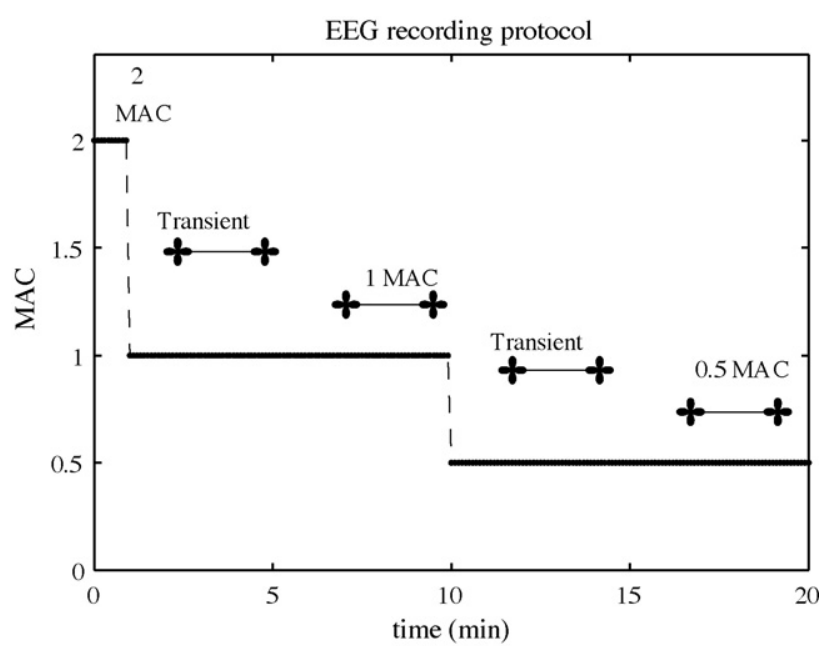

Fig. 1. Concentration of Desflurane changes stepwise (after intubation) from 2 to 1 and then to $0.5 \mathrm{MAC}$ in 20 minutes. EEG signal is recorded continuously, and BIS index is registered once in every minute.

of patients still move in response to a noxious stimulus. EEG recordings were obtained in the absence of any surgical stimulation. Fig. 1 illustrates the protocol that was followed to change the drug concentration. Desflurane concentration changed stepwise from $2 \mathrm{MAC}$ to $1 \mathrm{MAC}$ and then to $0.5 \mathrm{MAC}$ during 20 minutes. After each transition, the concentration was kept constant for about 10 minutes until the target drug concentration in the brain and EEG signals become stabilized. The surgery was started a few minutes after the increase of drug concentration following the twenty-minute EEG recording protocol. Sampling rates of EEG signals were equal to $400 \mathrm{~Hz}$, and $\mathrm{BIS}^{\mathrm{TM}}$ index was also recorded by the Aspect $\mathrm{XP}^{\mathrm{TM}}$ machine every minute, independently from the EEG recordings [13].

\subsection{Signal processing}

\subsubsection{Delta sub-bands and filtering}

POM and SOM can be defined for any pair of EEG wave components. In this paper, the higher-frequency component always belongs to [8-13] $\mathrm{Hz}$ frequency interval (which is referred to as alpha component), whereas the lower-frequency component may correspond to various delta sub-bands. In brief, delta sub-bands considered in this study are categorized to one of the following types: very slow $\delta^{V S}$, slow $\delta^{S}$, fast $\delta^{F}$, narrow $\left(\delta_{f}^{N}\right)$, cumulative slow1 $\left(\delta_{f}^{C S 1}\right)$, cumulative slow2 $\left(\delta_{f}^{C S 2}\right)$ and cumulative fast $\left(\delta_{f}^{C F}\right) . f$-indexed symbols indicate a set of delta sub-bands; for example, $\left(\delta_{f}^{C S 1}\right)$ consists of 25 delta sub-bands. EEG component corresponding to each of the above delta subbands was extracted from raw EEG using band-pass elliptic IIR filters (two cascaded high-pass and low-pass filters) provided by MATLAB ${ }^{\circledR}$ (ellip function). Orders of the filters were determined by ellipord function. We set peak-to-peak ripples (Rp) and minimum required stop-band attenuations (Rs) of the filters to 0.5 and $20 \mathrm{~dB}$, respectively. In the following, characteristics of the pass-band filters are described for all the abovementioned delta sub-bands.

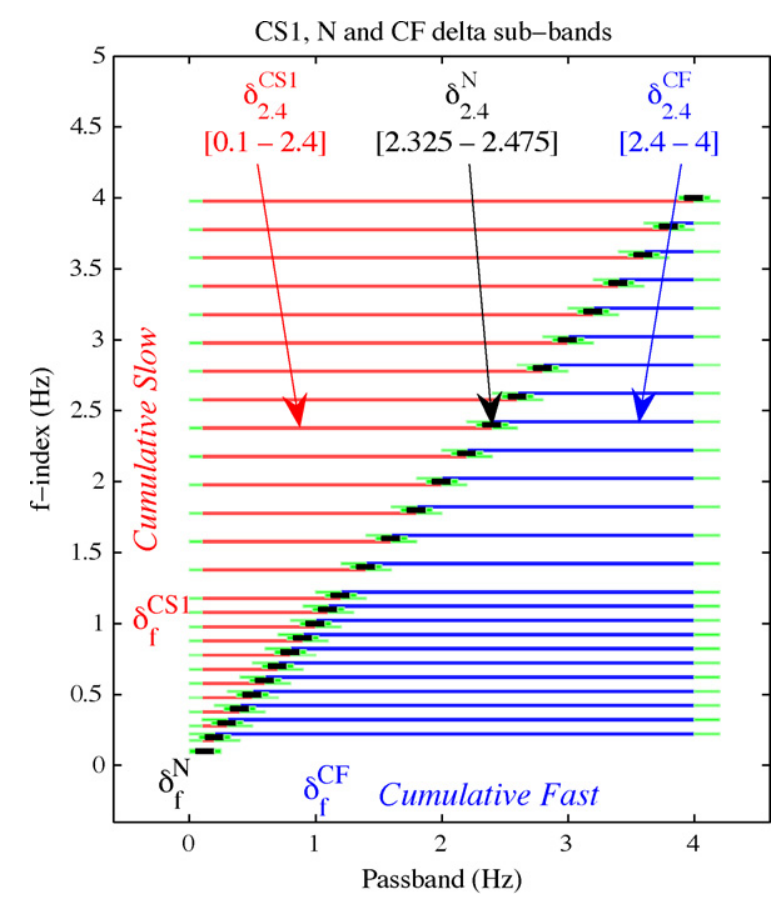

Fig. 2. A schematic representation of cumulative slow1 (CS1), narrow (N) and cumulative fast (CF) delta sub-bands. Transitions between pass-bands and stopbands of delta sub-bands are indicated by green rectangles in both end sides of pass-band horizontal bars. Since $\left(\delta_{f}^{C S 1}\right)$ and $\left(\delta_{f}^{C S 2}\right)$ sub-bands are only different in their lower edge frequencies (i.e. $0.1 \mathrm{~Hz}$ vs. $0.6 \mathrm{~Hz}),\left(\delta_{f}^{C S 2}\right)$ sub-bands are not shown in this figure. Pass-band intervals of three filters have been indicated in the figure. For example, $\delta_{2.4}^{N}$ is characterized by $[2.325-2.475] \mathrm{Hz}$ pass-band interval (width: $0.15 \mathrm{~Hz}$ ) and $0.05 \mathrm{~Hz}$ margins.

The intervals $[0.1-0.5] \mathrm{Hz}$ (very slow delta), $[0.7-1.7] \mathrm{Hz}$ (slow delta) and [2-4] Hz (fast delta) define $\delta^{V S}, \delta^{S}$ and $\delta^{F}$ sub-bands, respectively. Each of $\left(\delta_{f}^{C S 1}\right),\left(\delta_{f}^{C S 2}\right),\left(\delta_{f}^{N}\right)$ and $\left(\delta_{f}^{C F}\right)$ represents a set of delta sub-bands (Fig. 2). $\left(\delta_{f}^{C S 1}\right)$ and $\left(\delta_{f}^{C S 2}\right)$ consist of 25 and 19 sub-bands, respectively. Lower edge frequencies of these two sets of sub-bands are set to 0.1 and $0.6 \mathrm{~Hz}$, respectively. Upper edge frequencies increase linearly. A sub-band is thus determined by setting $f$ to a real number. For example, sub-bands $\delta_{2.4}^{C S 1}$ and $\delta_{2.4}^{C S 2}$ correspond to intervals $[0.1-2.4] \mathrm{Hz}$ and $[0.6-2.4] \mathrm{Hz}$, respectively. Lower and upper transition bands between pass-bands and stop-bands are equal to 0.09 and $0.2 \mathrm{~Hz}$, respectively. One major difference between $\left(\delta_{f}^{C S 1}\right)$ and $\left(\delta_{f}^{C S 2}\right)$ is that the first set gathers sub-bands relating to very slow, slow and fast delta activities, but the second one includes sub-bands relating only to slow and fast delta activities.

$\left(\delta_{f}^{N}\right)$ contains 26 narrow bands. Bandwidths are equal to $0.15 \mathrm{~Hz}$ and central frequencies increase linearly. Setting $f$ to a real number fully determines a sub-band. For example, $\delta_{2.4}^{N}$ corresponds to the sub-band [2.325-2.475] Hz. Transitions bands are set to $0.05 \mathrm{~Hz}$. $\left(\delta_{f}^{C F}\right)$ contains $24 \mathrm{sub}-$ bands. Lower edge frequencies of these sub-bands increase linearly while their upper edge frequencies are all equal to $4 \mathrm{~Hz}$. Transition bands are set to $0.2 \mathrm{~Hz}$. Setting the subscript $f$ to a given value specifies a unique sub-band; for example, $\delta_{2.4}^{C F}$ designate the sub-band [2.4 - 4] Hz. Fig. 2 illustrates the three $\left(\delta_{f}^{N}\right),\left(\delta_{f}^{C S 1}\right)$ and $\left(\delta_{f}^{C F}\right)$ sub-band sets. 
In order to better investigate the relation between slow delta band activities and alpha activities, distributions of central frequencies of $\left(\delta_{f}^{C S 1}\right),\left(\delta_{f}^{C S 2}\right),\left(\delta_{f}^{N}\right)$ and $\left(\delta_{f}^{C F}\right)$ sets are more concentrated in lower delta band (i.e. $<1.2 \mathrm{~Hz}$ ).

Throughout this paper, if a parameter is related to one of the above-mentioned sub-bands, the name of that parameter is concatenated with the superscript of the given sub-band. For example, if a POM is calculated between a slow delta sub-band $\left(\delta^{S}\right)$ and alpha band, it is then denoted by S-POM. Similarly, other parameters such as CS1-POM, N-SOM, CF-POM, etc. are also defined.

\subsubsection{Calculating $P O M$}

The procedure for calculating POM between an alpha wave and an EEG wave component in a given delta sub-band is as follows. For the sake of simplicity, we use the term delta wave instead of delta sub-band wave.
The EEG signal is down sampled to $80 \mathrm{~Hz}$, decomposed into $50 \%$ overlapped epochs of $30 \mathrm{sec}$ and then the mean value is removed from each epoch. Fig. 3(a) illustrates $10 \mathrm{sec}$ of an EEG epoch recorded at $1 \mathrm{MAC}$.

Delta $\delta(t)$ and alpha $\alpha(t)$ waves are extracted from the EEG epoch (IIR filtering) and their powers are normalized to one (Fig. 3[b1, b2, d]). Two different examples of $\delta(t)$ are illustrated in the figure. In Fig. 3(b1), $\delta(t)$ represents slow delta sub-band $\delta^{S}$, and in Fig. 3(b2), it represents fast delta sub-band $\delta^{F}$.

Analytic signal of a given delta wave is obtained by taking Hilbert transform of $\delta(t)[14,15]$ :

$$
\delta_{\text {Anal }}(t)=\delta(t)+i \mathbb{H}(\delta(t))
$$

$$
\begin{aligned}
\mathbb{H}(\delta(t)) & =-\frac{1}{\pi} \lim _{\varepsilon \rightarrow 0} \int_{\varepsilon}^{\infty} \frac{\delta(t+\tau)-\delta(t-\tau)}{\tau} d \tau \\
& =F^{-1}(-i \operatorname{sgn}(\omega) F(\delta(t)))
\end{aligned}
$$

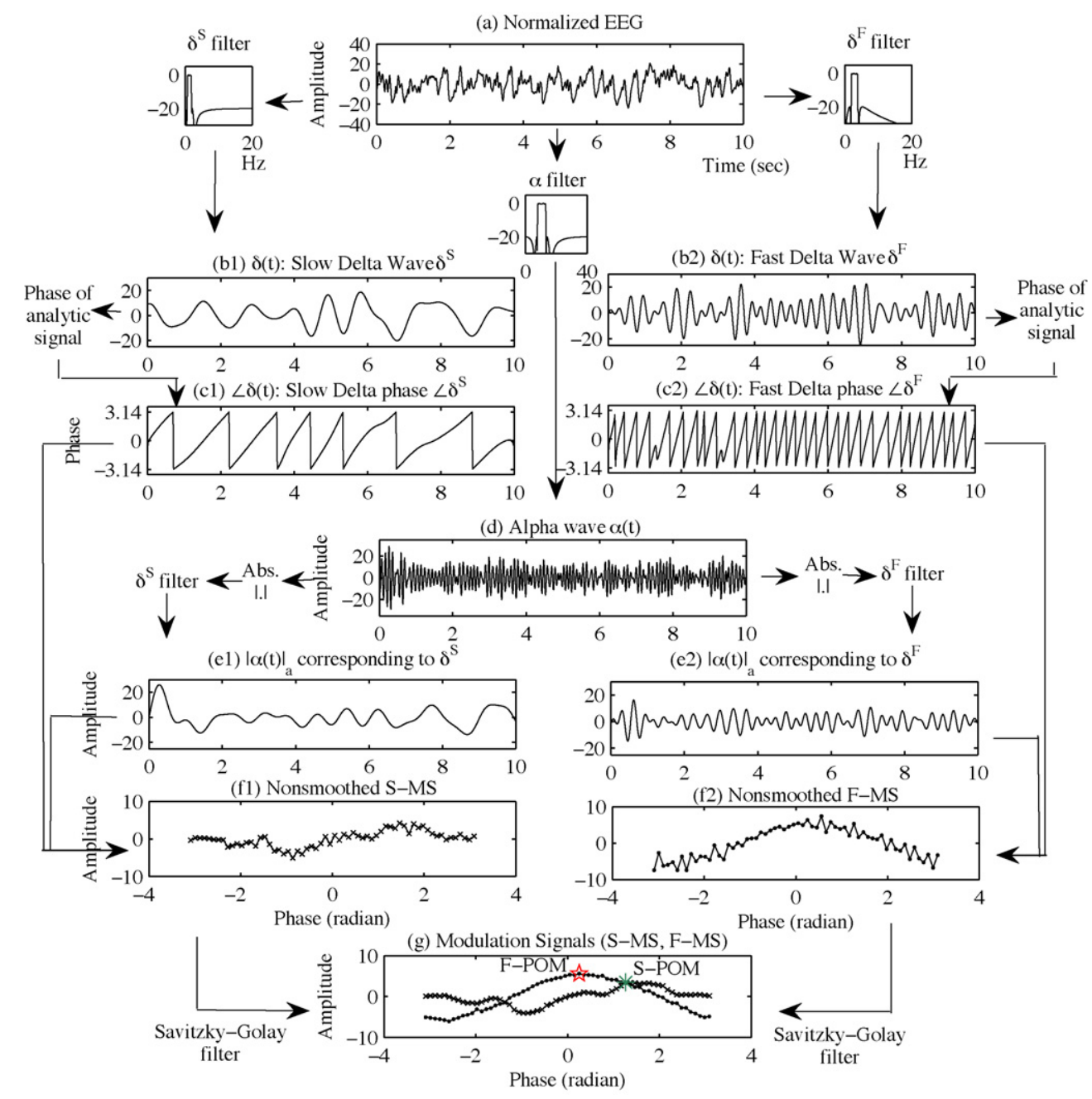

Fig. 3. (a) $10 \mathrm{sec}$ of a zero-mean normalized EEG epoch at 1 MAC Desflurane anesthesia. (b) Delta waves extracted from the EEG epoch. Delta waves represent slow and fast delta sub-bands in part (b1) and (b2), respectively. Frequency responses of IIR filters corresponding to slow and fast delta sub-bands are also sketched in the figure. (c) Delta phases corresponding to delta waves illustrated in part (b). (d) Alpha component of the EEG epoch. Pass-band of the alpha-band filter is in [8-13] Hz interval. (e) Alpha-amplitude signals corresponding to slow and fast delta waves. They are obtained by taking the absolute values of alpha waves and applying a filtering procedure. Slow and fast delta sub-band IIR filters have been employed for alpha-amplitude signals illustrated in (e1) and (e2), respectively. (f) Non-smoothed modulation signals. Notice that non-smoothed modulation signals are obtained by analyzing the entire $30 \mathrm{~s}$ of EEG epoch. (g) Modulation signals resulting after applying Savitzky-Golay filters on non-smoothed modulation signals (f1, f2). S-POM and F-POM are indicated by two markers. 


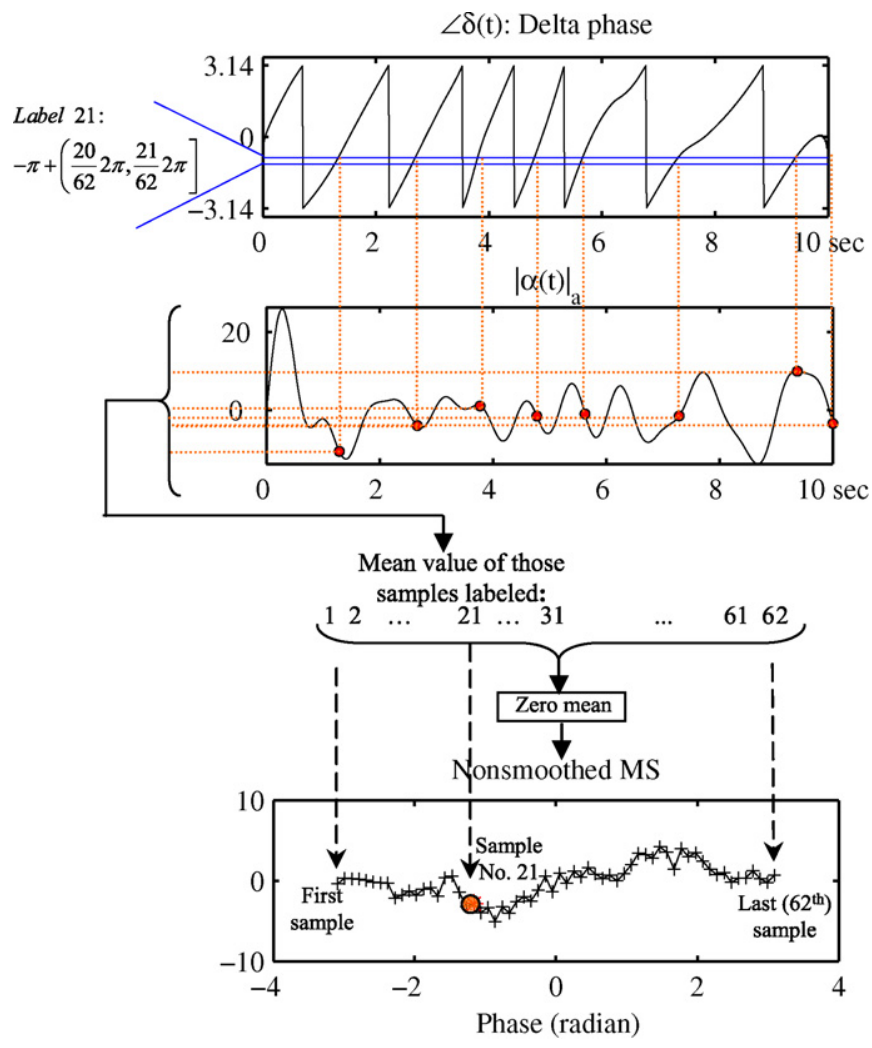

Fig. 4. A schematic diagram of the procedure for obtaining a 62-point modulation signal. Concurrent samples of alpha-amplitude $|\alpha|_{a}$ and delta phase $\angle \delta$ signals take same labels. For instance, those samples of delta phase signal that are labeled 21 are indicated in the diagram. These samples are between -1.1148 and $-1.0134 \mathrm{rad}$. Mean value of alpha-amplitude signal is calculated for each labe (between 1 and 62). The resulted 62-point signal is then centered to form the non-smoothed modulation signal.

where $\mathbb{H}, F$ are Hilbert and Fourier transforms, respectively, $i=$ $\sqrt{-1}$ and $\operatorname{sgn}(\omega)$ is sign function which takes -1 and +1 values for negative and positive arguments, respectively.

The angle of the obtained analytic signal $\delta_{\text {Anal }}(t)$ represents instantaneous phase of delta wave. This angle is calculated as follows (Fig. 3[c1, c2]).

$\angle \delta(t)=\tan ^{-1}(\mathbb{H}(\delta(t)) / \delta(t))$

Basically, it can be said that each cycle of the delta wave is mapped to the $(-\pi, \pi)$ interval.

Absolute value of $\alpha(t)$ is passed through the same IIR filter that was employed to extract $\delta(t)$ from the EEG epoch and the power of the resulted signal is normalized to one. This resulted signal is referred to as alpha-amplitude signal and is denoted by $|\alpha(t)|_{a}$ (Fig. 3(e1, e2))

A 62-bin amplitude-distribution is formed by data samples of $|\alpha(t)|_{a}$ according to data samples of $\angle \delta(t)(\mathrm{Fig} .3(\mathrm{f} 1, \mathrm{f} 2))$. This 62-point amplitude-distribution, which is referred to as modulation signal (MS), indicates mean amplitude of alpha waves for different delta phases. The detailed procedure for calculating an MS is as follows (this procedure is illustrated schematically in Fig. 4):
- the $(-\pi, \pi]$ interval is divided into 62 bins $(\sim 0.1 \mathrm{rad}$ for each bin);

- each data sample of $\angle \delta(t)$ is labeled with a number between 1 and 62 (bin index) according to its value. For example, Fig. 4 indicates that the bin index 21 corresponds to the $(-1.1148$, -1.0134] rad phase interval;

- concurrent data samples of $|\alpha(t)|_{a}$ and $\angle \delta(t)$ take the same bin index;

- a 62-bin amplitude-distribution is formed using data samples of $|\alpha(t)|_{a}$. To do so, the mean amplitude of those data samples of $|\alpha(t)|_{a}$ having the same label is calculated.

Once the 62-point amplitude-distribution is obtained, the mean value of the amplitude-distribution is removed and the resulted 62-point signal is then smoothed using a SavitzkyGolay filter [16]. Savitzky-Golay is an FIR filter that performs a local polynomial regression (of degree $\mathrm{k}$ ). The polynomial order and frame size of the Savitzky-Golay filter were set to 3 and 19 , respectively (sgolayfilt function in MATLAB ${ }^{\circledR}$ ). The smoothed 62-point signal is called modulation signal MS. (Fig. 3(g)).

POM is the phase for which the MS is maximal: $\mathrm{POM}=$ arg $\max (\mathrm{MS})$ (see markers in Fig. 3(g)). If necessary, an $\theta \in(-\pi, \pi]$ unwrapping procedure can be used to ensure phase continuity in successive EEG epochs.

\subsubsection{Calculating SOM}

SOM is the other parameter that will be discussed beside POM in this study. SOM may accompany POM to indicate to what extent the modulation between the considered delta wave and alpha band is strong. A strong modulation means coincidence of a fixed delta phase value with local maximums (or minimums) of $|\alpha(t)|_{a}$ in successive cycles of delta oscillations. Conversely, If the modulation is weak, maximums (or minimums) of $|\alpha(t)|_{a}$ coincide with different delta phase values. Fig. 5 compares a strong and a weak amplitude modulation. This figure illustrates $15 \mathrm{sec}$ of two delta phase signals $\angle \delta(t)$ and their corresponding alpha-amplitude signals $|\alpha(t)|_{a}$. Maximum values of $|\alpha(t)|_{a}$ are connected to their corresponding delta phases by vertical lines. In part A, most of these vertical lines point to phase values around $2.5 \mathrm{rad}$ whereas, in part $\mathrm{B}$, vertical lines randomly target different phase values. As a consequence, MS of a weak modulation is flatter than MS of a strong modulation and it does not usually have a dominant extremum. SOM is a parameter, which is designed to quantify this characteristic of MS and to measure its flatness. In this study, the MS is smoothed, and then its standard deviation is expressed as SOM. Smoothing is done by the Savitzky-Golay filter used before. Smoothing increases the contrast between a weak and a strong modulation. As Fig. 5 depicts, the MS in part A has both a wide range and a wide histogram and thus it has a high standard deviation (4.87 vs. 0.95 in part B).

\subsubsection{Monitoring POM and SOM}

Fig. 6 part A illustrates a two-dimensional view of MSs of a patient obtained during the twenty-minute EEG record- 
(A) Strong Modulation

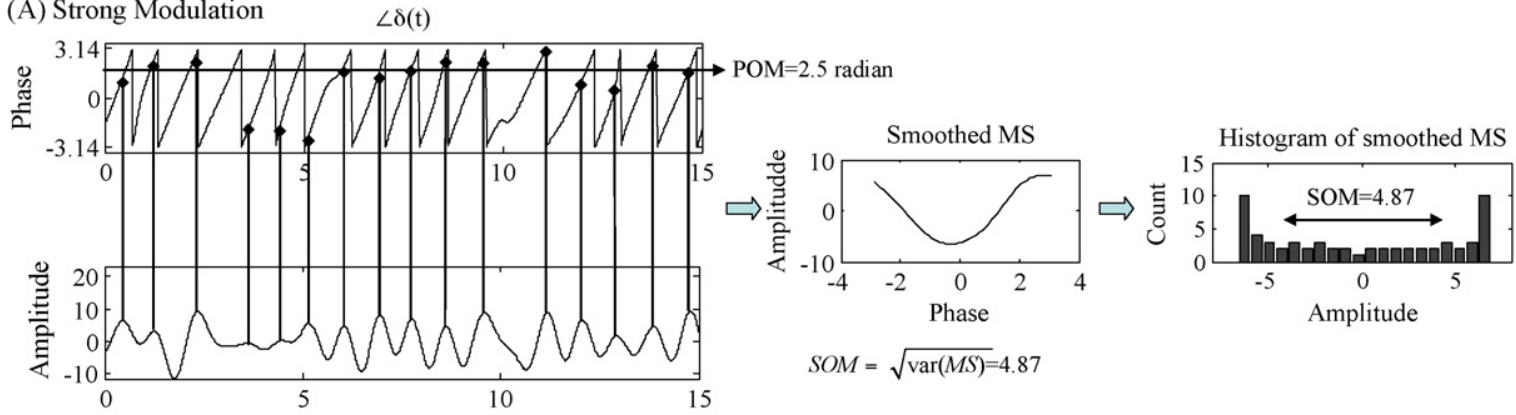

(B) Weak Modulation
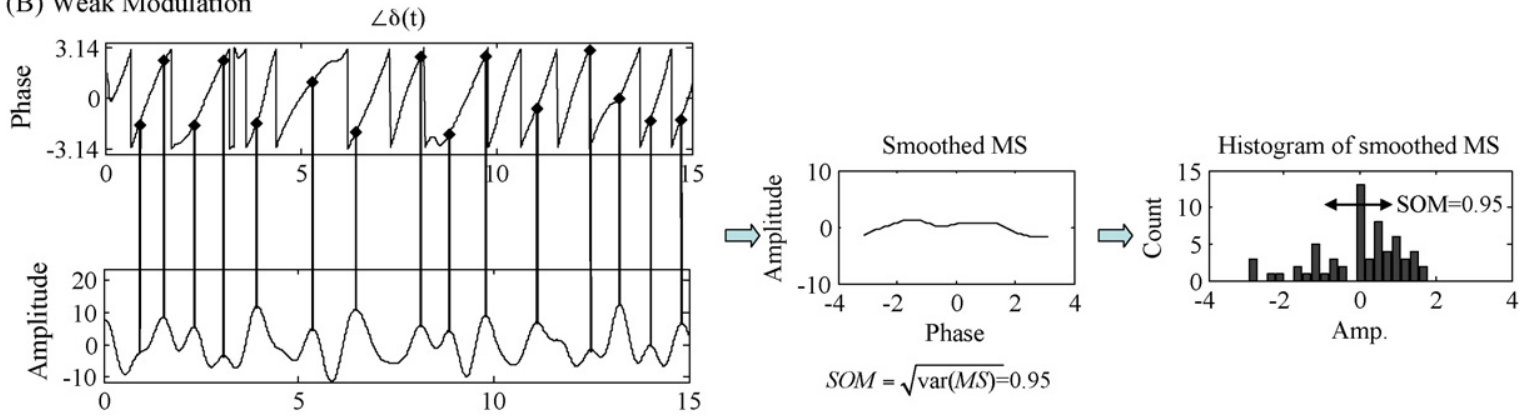

Fig. 5. Comparison of a strong (A) and a weak (B) modulation. (Left) $15 \mathrm{sec}$ of two delta phase signals $\angle \delta$ and their corresponding alpha-amplitude signals $|\alpha|_{a}$. Maximum values of $|\alpha|_{a}$ are connected to their corresponding delta phases by vertical lines. In part A, most of the vertical lines point to phase values around 2.5 rad. In part B, vertical lines do not point to a specific phase value. (Middle) Smoothed modulation signals. The amplitude of the smoothed MS in part A is higher than the smoothed MS in part B. It also contains dominant local extrema, while the smoothed MS in part B has not a dominant extremum. (Right) Histograms of smoothed modulation signals. The histogram in part A is wider than the histogram in part B. SOM, which is the standard deviation of smoothed MS, is higher in part A than in part B.

ing. The left and right side images are related to S-MSs and F-MSs, respectively. Each column of the images, which are called Modulation Images (MI), corresponds to an SMS or F-MS at a given time. As indicated in Fig. 6 parts $\mathrm{B}$ and $\mathrm{C}, \delta^{S}-$ and $\delta^{F}$-related POM and SOM parameters can be monitored continuously from their corresponding S-MI and F-MI. Since it is generally expected that POM values do not change rapidly in successive EEG epochs, a smoothing filter (Savitzky-Golay filter, order 3, frame size 9) is applied on MI in the time-axis direction.

POM monitoring indicates that DOA is correlated to both S-POM and F-POM. In other words, in different DOA, maximum (or minimum) amplitudes of alpha waves appear in different phases of slow and fast delta waves. It can be said that delta waves and fast EEG oscillations are two phase-locked brain activities, and that their phase differences change with DOA. However, these phase differences are not identical for slow and fast delta waves. For instance, in this patient, when Desflurane concentration is stabilized at $1 \mathrm{MAC}, \mathrm{F}-\mathrm{POM}$ and S-POM are around 0.5 and $2 \mathrm{rad}$, respectively. The maximum range of S-POM values over the entire twenty-minute EEG recordings is greater than the maximum range of F-POM values. In deep anesthesia (2 MAC) S-POM and F-POM are around $-0.5 \mathrm{rad}$, and in light anesthesia (0.5 MAC), the S-POM is around $\pi \mathrm{rad}$, whereas F-POM is around 2 rad. F-POM has a regular upward trend when DOA decreases, while S-POM does not exhibit such a regular tendency.

\section{Results}

\subsection{Fast EEG oscillations are modulated by very slow, slow and fast delta waves}

We derived $\delta_{f}^{N}$ - related SOMs (N-SOM) in different stages of anesthesia to find the relation between different narrowband delta sub-band components and alpha waves. Fig. 7 depicts medians and quartile ranges (i.e. error bars at 25 and 75 percentiles: prctile function in MATLAB ${ }^{\circledR}$ ) of N-SOM values obtained in all patients. These values are illustrated versus central frequencies of $\delta_{f}^{N}$-sub-bands (i.e. $f$-index) at $t=0,9$ and 19 minutes. These time indices are related to different stabilized depths of anesthesia at 2,1 and 0.5 MAC, respectively. Medians and error bars indicate that delta band can be divided into three regions in which $\mathrm{N}-\mathrm{SOM}$ values reach a maximum. This indicates that alpha waves are basically modulated by three delta sub-bands and also points out that the modulation between delta waves and alpha waves is not only seen in non-REM sleep [2] but also during various DOA.

Here, we will refer to the frequency interval $[0.1-0.5] \mathrm{Hz}$ as "very slow" delta sub-band. This frequency interval, which is the lowest delta sub-band, appears to be independent from Desflurane concentration. The very slow delta sub-band may correspond to the slow delta activity that has been reported in [17-19]. The second delta sub-band is referred to as "slow" delta sub-band. Desflurane concentration changes the boundary of this sub-band. In deep anesthesia at $2 \mathrm{MAC}$, it is almost extended from 0.5 to $1.2 \mathrm{~Hz}$. When DOA decreases, this region moves 
(A)

(a1) S-MI

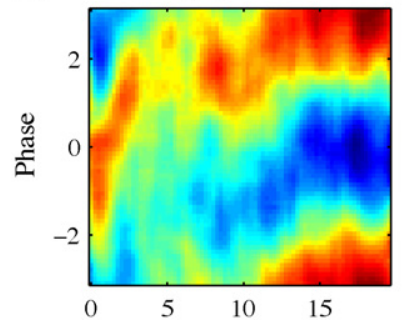

(B)

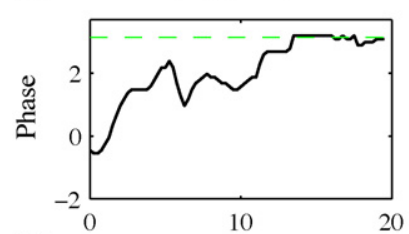

(C)

(c1) S-SOM

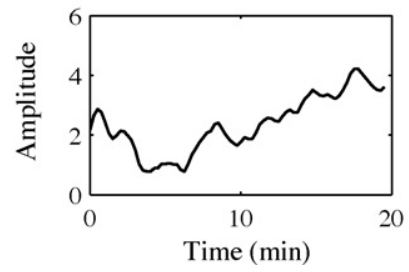

(a2) F-MI

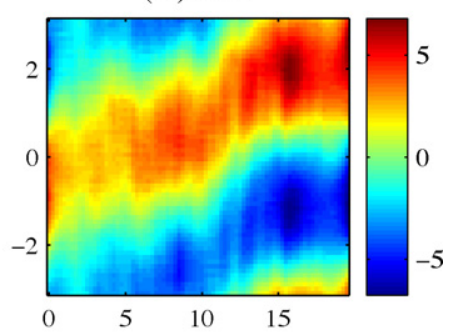

(b2) F-POM

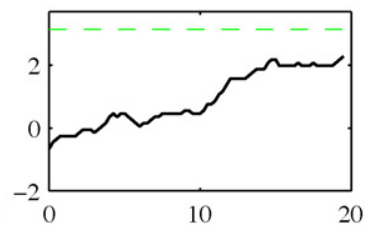

(c2) F-SOM

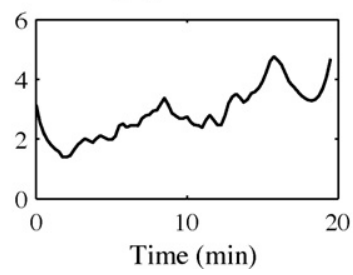

Fig. 6. (A) Modulation images corresponding to slow (a1) and fast (a2) delta sub-bands obtained on a patient. Each column of S-MI or F-MI corresponds to a modulation signal at a given time. (A) POM values in time domain. SPOM and F-POM values increase with a decrease in Desflurane concentration. Maximum range of S-POM during the entire $20 \mathrm{~min}$ of data analysis is higher than the maximum range of F-POM. In light anesthesia, S-POM is around $+\pi$ rad (green dashed line). (C) S-SOM and F-SOM signals indicate to what extent the modulation is strong, and thus to what extent POM signals are reliable.

a bit toward higher frequencies. For example at $0.5 \mathrm{MAC}$ it is extended from 0.6 to $1.8 \mathrm{~Hz}$. The central frequency of the slow delta band is around $1 \mathrm{~Hz}$ at $1 \mathrm{MAC}$ and an increase/decrease of drug concentration may shift, a little bit, this frequency towards a lower/higher value. Fast delta band is the third sub-band that can be distinguished by N-SOM data. Basically, this band includes frequencies higher than $2 \mathrm{~Hz}$. Central frequency of fast delta band varies with Desflurane concentration. However, it is roughly around $2.8 \mathrm{~Hz}$. We mention that the division of delta band into VS, S and F delta sub-bands (introduced in section 2.2.1) has been done mainly based on N-SOM results relating to moderate and light anesthesia (1 and 0.5 MAC).

\subsection{POM and SOM in slow and fast delta waves}

In the case reported in section 2.2.4, it was shown that POM and SOM evolve with anesthesia. It was also indicated that slow and fast delta sub-bands give different POM values at a same given MAC. In this section, the overall characteristics of $\delta^{S}-$ and $\delta^{F}$ - related POM and SOM are reported based on 10 patients.

Error bars in Fig. 8(a) illustrate mean and standard deviation values of S-POM and F-POM parameters in the patients in every minute of EEG recording protocol. Dot and X markers correspond to S-POM and F-POM parameters, respectively. These markers indicate that both types of POMs increase with

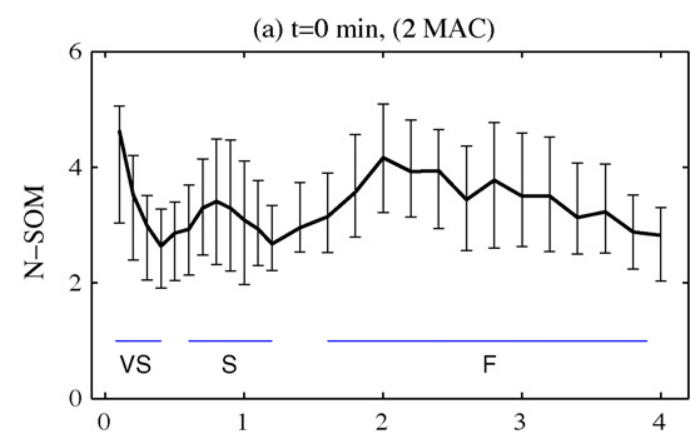

(b) $\mathrm{t}=9 \mathrm{~min},(1 \mathrm{MAC})$

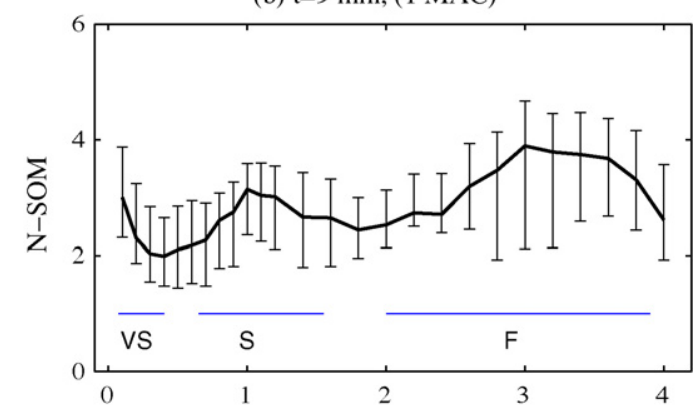

(c) $\mathrm{t}=19 \mathrm{~min},(0.5 \mathrm{MAC})$

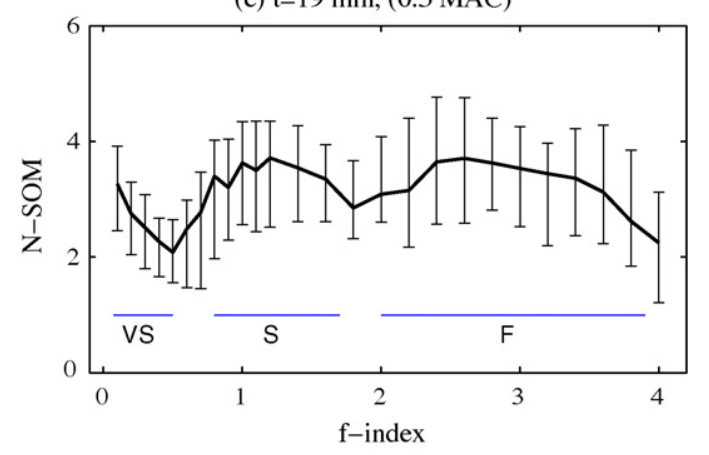

Fig. 7. Median and quartile ranges of SOM values corresponding to narrow delta sub-bands (N-SOM) in all patients in different stabilized anesthesia in deep $(t=0 \mathrm{~min}, 2 \mathrm{MAC})$, moderate $(t=9 \mathrm{~min}, 1 \mathrm{MAC})$ and light anesthesia $(t=19 \mathrm{~min}$, $0.5 \mathrm{MAC})$. Error bars roughly indicate three dominant delta sub-band frequency intervals: $[0.1-0.5] \mathrm{Hz},[0.7-1.7] \mathrm{Hz}$ and $[2-4] \mathrm{Hz}$ relating to very slow (VS), slow (S) and fast (F) delta sub-bands, respectively.

the level of consciousness. However, the way that they increase depends on the type of delta sub-band. For example, at $2 \mathrm{MAC}$ $(t=0)$, mean values of S-POM and F-POM are around $-0.5 \mathrm{rad}$, and at $1 \mathrm{MAC}(t=9 \mathrm{~min})$, these values are about 1.6 and $0.3 \mathrm{rad}$ respectively. In light anesthesia $(t=19)$ standard deviation of $\mathrm{S}$-POM is less than the standard deviation of F-POM. In deep anesthesia $(t=0)$, it is the F-POM parameter that has a lower standard deviation.

Fig. 8(b) illustrates mean and standard deviation of S-SOM and F-SOM parameters in the patients. Dot and X markers correspond to S-SOM and F-SOM parameters. A comparison between mean values of S-SOMs and F-SOMs at $t=0$ reveals that in deep anesthesia the relation between slow delta sub-band and alpha band is weak compared with the relation between fast delta subband and alpha band. Conversely, in light anesthesia, slow delta has a stronger relation to the alpha band. 
(a) S-POM, F-POM

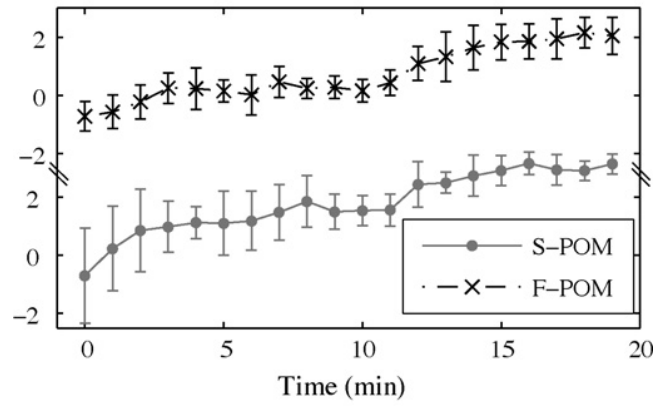

(b) S-SOM, F-SOM

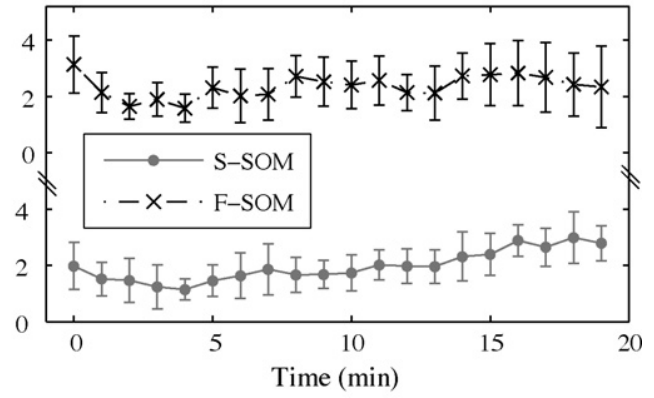

Fig. 8. Mean and standard deviation of S-POM and F-POM (part a) and S-SOM and F-SOM (part b) in all patients in every minute of EEG recording. When DOA reduces, S-POM and F-POM increase. The increase is more for S-POM than for F-POM. F-SOM has a biphasic response to each rapid change of anesthetic drug concentration. S-SOM becomes minimal in response to the first rapid change of drug concentration (from 2 to $1 \mathrm{MAC}$ ) and then increases with reduction of Desflurane concentration.

F-SOM values have a rather complex behavior when Desflurane concentration changes from 2 to $1 \mathrm{MAC}$ and from 1 to 0.5 MAC. Decreasing MAC induces oscillations in F-SOM values. These oscillations consist of a reduction and an increase of F-SOM values (like a biphasic response). More precisely saying, F-SOM exhibits a minimum about 3 minutes after the transition from 2 to 1 MAC. This behavior is also seen, with a lesser degree, about 2 minutes after the transition from 1 to 0.5 MAC. F-SOM exhibits maximum values about 5 minutes following transitions from 2 to 1 and from 1 to $0.5 \mathrm{MAC}$.

For S-SOM parameter, we could not find an identical biphasic behavior in different patients; therefore, the mean value of S-SOM does not indicate a meaningful biphasic response. SSOM becomes minimal in response to the first rapid change of drug concentration (from 2 to $1 \mathrm{MAC}$ ) and then increases with reduction of drug concentration especially after the transition from 1 to $0.5 \mathrm{MAC}$.

\subsection{Different delta sub-bands and their relating POM: Application to DOA monitoring}

In section 3.2, it was pointed out that POM values change with DOA. This suggests that POM may be used for determining DOA. In order to determine DOA with POM data, it is necessary to study the characteristics of POM relating to different delta sub-bands in different DOA to find a proper delta sub-band for this purpose. To do so, we consider the three delta sub-band sets $\left(\delta_{f}^{C S 1}\right),\left(\delta_{f}^{C S 2}\right)$ and $\left(\delta_{f}^{C F}\right)$ and compare their relat- ing POMs and SOMs as indicated in Fig. 9. This figure consists of three columns and three rows. The first row is related to results obtained from $\left(\delta_{f}^{C S 1}\right)$ set. The second and third rows are related to $\left(\delta_{f}^{C S 2}\right)$ and $\left(\delta_{f}^{C F}\right)$ set, respectively. In the first column of Fig. 9, mean POM values are illustrated in different depths of anesthesia at $t=0,5,9,15$ and 19 minutes. For the sake of simplicity, these time indexes, which are related to stabilized anesthesia at 2, 1 and $0.5 \mathrm{MAC}$ and the transitions, are referred to as stage 1, stage 2 and so on, respectively. For each given stage and $f$-index, mean value of POMs in all patients is obtained at that given time and the subsequent $30 \mathrm{sec}$ (i.e. over three subsequent EEG epochs). The second column of Fig. 9 is related to inter-patient variability of POM values. In order to quantify this variability, the standard deviation of POM values is calculated for each given stage and $f$-index. As before, each standard deviation value is calculated over 3 successive EEG epochs. Finally, the last column in Fig. 9 is related to mean SOM values for the given stage and $f$-indexes.

It can be inferred from Fig. 9(a1) that those $\left(\delta_{f}^{C S 1}\right)$ subbands with upper edge frequencies less than about $1.5 \mathrm{~Hz}$ (i.e. $\delta_{f<1.5}^{C S 1}$ ), cannot distinguish well different stages of anesthesia at $t=0,5,9,15$ and 19 minutes. Standard deviation of POM values in Fig. 9(a2) also indicates that inter-patient variability of $\left(\delta_{f<1.5}^{C S 1}\right)$ delta sub-bands are high especially from deep to moderate anesthesia. An increase in $f$-index from 1.5 to $4 \mathrm{~Hz}$ reduces the inter-patient variability of POM values in all stages of anesthesia. Reduction of inter-patient variability of POM values with $f$-index raises the following questions: 1) Are fast delta waves better than slow waves for quantifying DOA?, 2) Might it be due to interaction between very slow and slow delta activities that standard deviation of CS1-POMs are high in low-value $f$-indexes?

$\left(\delta_{f}^{C S 2}\right)$ and $\left(\delta_{f}^{C F}\right)$ sub-bands have been designed to answer these questions. In $\left(\delta_{f}^{C S 2}\right)$ sub-bands, very slow activities have been excluded from EEG activities and in $\left(\delta_{f}^{C F}\right)$ sub-bands, different formation of fast delta bands (with and without including slow delta band) is considered for being analyzed.

A comparison between Fig. 9(b1, b2) and Fig. 9(a1, a2) reveals that discarding the very slow delta activities not only enhances the contrast between different stages of anesthesia, but also reduces the inter-patient variability of POM values. For example, for an $f$-index which is around $1 \mathrm{~Hz}$, mean CS2POM values in stages 1 and 2 are almost equal to -0.7 and $1.7 \mathrm{rad}$ respectively, while these values change to 0.2 and $1.2 \mathrm{rad}$, respectively, for $\delta_{\simeq 1}^{C S 1}$. The inter-patient variability of CS2-POM is also lower than the inter-patient variability of CS1-POM in all stages of anesthesia when $f$-index is equal to $1 \mathrm{~Hz}$.

One interesting point in Fig. 9(b2) is that the increase of $f$ index beyond $2 \mathrm{~Hz}$, does not always reduce standard deviation of CS2-POM in stages 4 and 5 (notice the slight increase of std curve in stage 5). This may be an indication that fast delta activities may have inferior performances (in terms of standard deviation) than slow delta activities for determining DOA in light anesthesia (this is the case that was indicated in Fig. 8). As a complementary viewpoint, mean values of CS2-SOM parameter in Fig. 9(b3) also show that the performance of slow delta sub- 


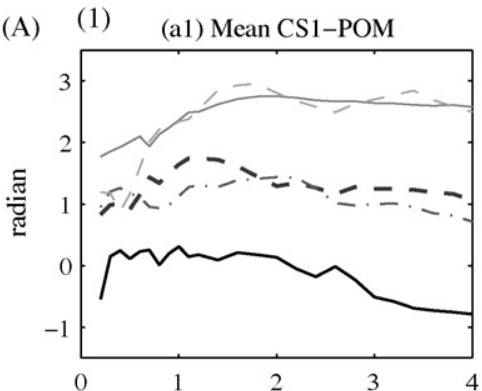

(2)

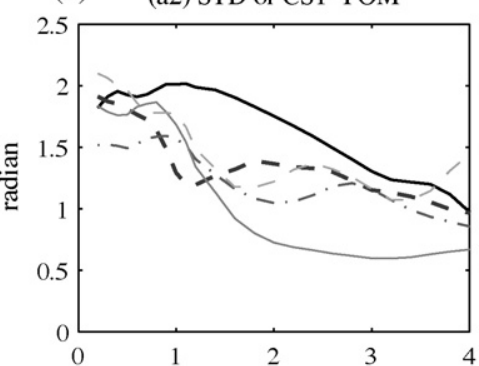

(B)

(b1) Mean CS2-POM

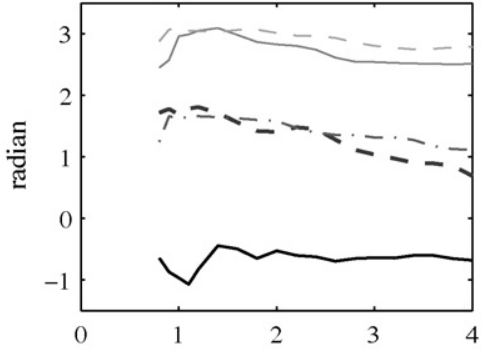

(C)

(c1) Mean CF-POM

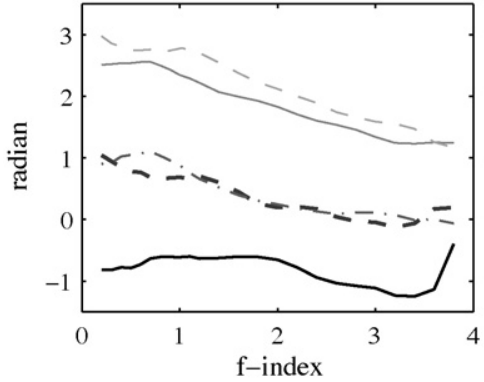

(b2) STD of CS2-POM

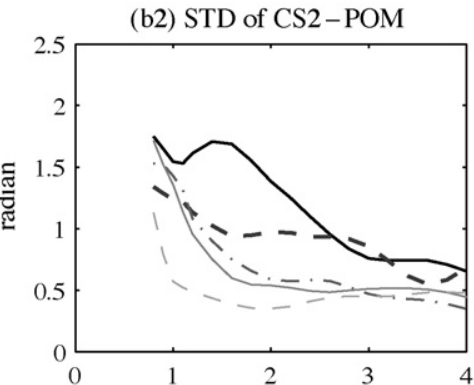

(c2) STD of CF- POM

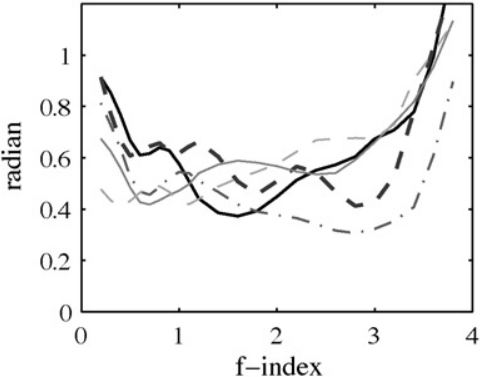

(3)

(a3) Mean CS1-SOM

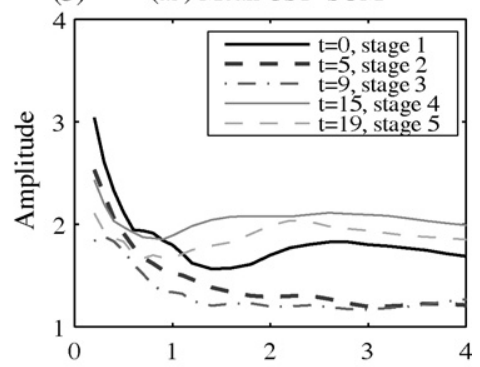

(b3) Mean CS2-SOM

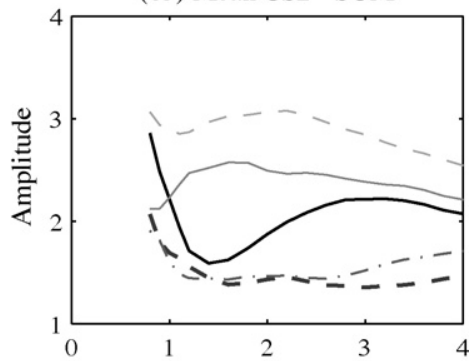

(c3) Mean CF-SOM

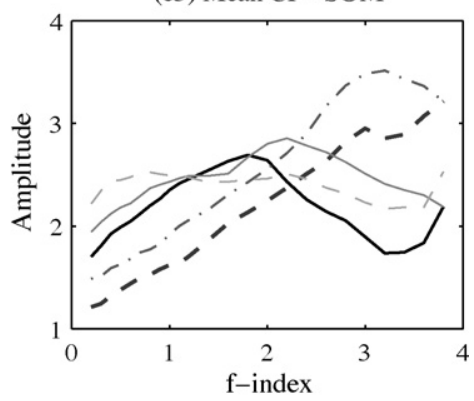

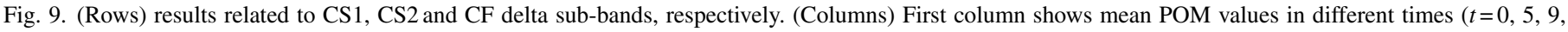

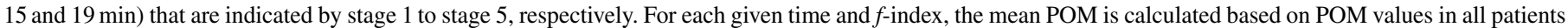

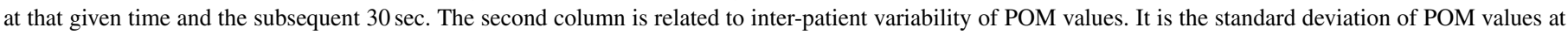
each given time and $f$-index. The last column is related to mean SOM values.

bands can be better than the performance of fast delta sub-bands in light anesthesia because for those $f$-indexes greater than about $2 \mathrm{~Hz}, \mathrm{SOM}$ values start to decrease in stages 4 and 5 .

According to abovementioned observations from Fig. 9, it can be inferred that concatenations of faster delta sub-bands with slower delta sub-band (i.e. increasing the $f$-index in $\left(\delta_{f}^{C S 2}\right)$ subbands) significantly improves the performance of determining DOA in deep and moderate anesthesia. However, in light anesthesia the performance is degraded a little bit. This indicates that basically, faster delta sub-bands must be better candidates for determining DOA than slower delta sub-bands. The structure of $\left(\delta_{f}^{C F}\right)$ sub-bands helps us to find out which combination of fast and slow delta sub-bands is reasonable for calculating POM and thus for determining DOA in different stages of anesthesia.

According to Fig. 9(c1) the performance of CF-POM for separating deep, moderate and light anesthesia is good for $f$-indexes smaller than about $3.5 \mathrm{~Hz}$. In terms of inter-patient variability of CF-POM values (Fig. 9(c2)), results are not very good for $f$-indexes greater than 2.5 or less than $0.5 \mathrm{~Hz}$. More precisely saying, an $f$-index, which is greater than $2.5 \mathrm{~Hz}$, fails to give low standard deviation in light and deep anesthesia (stages 1, 4, 5 ), and an $f$-index which is less than $0.5 \mathrm{~Hz}$, basically fails to yield a low standard deviation in deep and moderate anesthesia (stages 1 to 3). Fig. 9(c3) also confirms deductions derived from Fig. 9(c2). For a high value $f$-index, SOM values are low in light and deep anesthesia, and for a low value $f$-index, SOM values are low in moderate and deep anesthesia. It seems that a tradeoff between these two extremes, which also yields a high SOM in deep anesthesia, would be $f$-indexes between 1.2 and $2 \mathrm{~Hz}$.

Fig. 10(a1-a3) illustrates POM signals of the patients for three delta sub-bands $\delta_{0.8}^{C F}, \delta_{1.8}^{C F}$ and $\delta_{2.8}^{C F}$. Thick lines are mean values of POM signals. These lines indicate that POM values increase monotonically with a decrease in DOA. Dispersion of POM signals show that the best performance of each sub-band (in terms of inter-patients variability) for determining DOA takes place in different stages of anesthesia as if they have complementary performances. In moderate anesthesia $\delta_{2.8}^{C F}$ - related POM (CF28-POM) has the best performance, and in deep and light anesthesia best performances are obtained by $\delta_{1.8}^{C F}$-related POM $(\mathrm{CF} 18-\mathrm{POM})$ and $\delta_{0.8}^{C F}$ - related POM (CF08-POM), respectively. This is what we expected from Fig. 9(c2). In fact, in this figure, minimum values of the illustrated curves appear in different $f$-indexes. In deep anesthesia (stage 1 ) the minimum is around $1.7 \mathrm{~Hz}$, in moderate anesthesia (stages 2,3) the minimum 
(a1) $\mathrm{CF}-\mathrm{POM} \mathrm{f}=0.8 \mathrm{~Hz}$

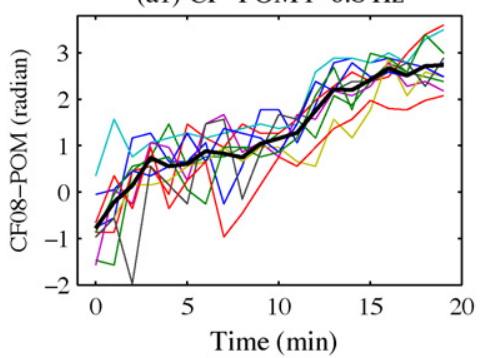

(b) BIS index

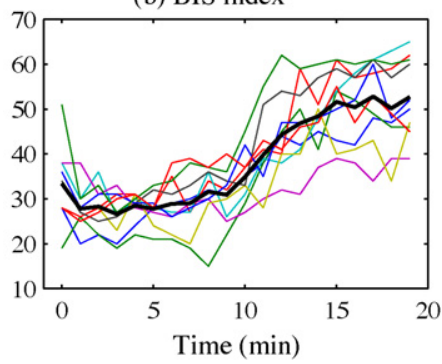

(a2) $\mathrm{CF}-\mathrm{POM} \mathrm{f}=1.8 \mathrm{~Hz}$

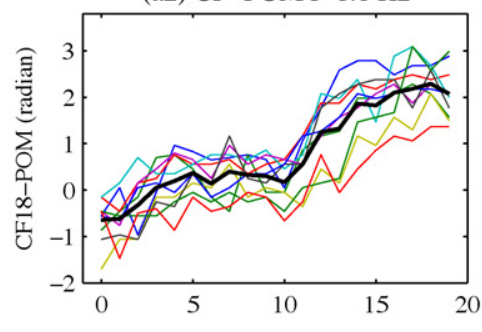

(a3) $\mathrm{CF}-\mathrm{POM} \mathrm{f}=2.8 \mathrm{~Hz}$

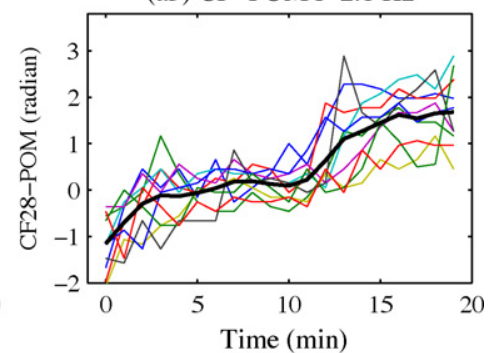

(c) Normalized-STD

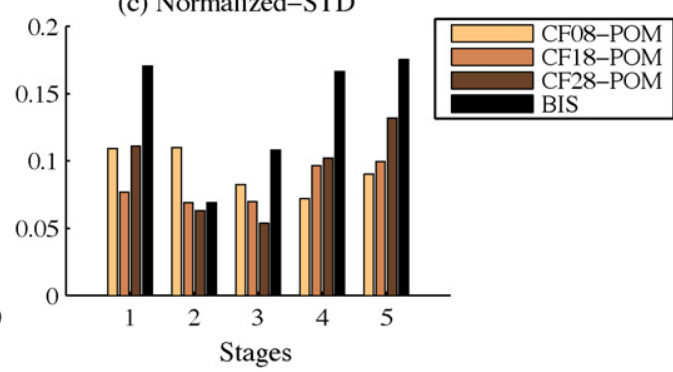

Fig. 10. (a1-a3) CF-POM values relating to $\delta_{0.8}^{C F}, \delta_{1.8}^{C F}$ and $\delta_{2.8}^{C F}$ (CF08-POM, CF18-POM and CF28-POM) obtained in all patients during the 20-minute EEG recording protocol. Thick lines indicate mean values of POMs. (b) BIS indexes and their mean value obtained in same patients with Aspect XP ${ }^{\mathrm{TM}}$ machine. (c) Normalized standard deviations of POM values relating to $\delta_{0.8}^{C F}, \delta_{1.8}^{C F}, \delta_{2.8}^{C F}$ delta sub-bands and BIS indexes in five stages of anesthesia at $t=0,5,9,15$ and 19 min.

is around $3 \mathrm{~Hz}$, and in light anesthesia (stages 4,5 ) the minimum is around $0.7 \mathrm{~Hz}$.

Fig. 10(b) illustrates BIS ${ }^{\mathrm{TM}}$ indexes and their mean values (thick line) in every minute of EEG recording. Inter-patient variability of BIS ${ }^{\mathrm{TM}}$ index is higher in light anesthesia than in deep and moderate anesthesia. In addition, there are some patients for whom BIS ${ }^{\mathrm{TM}}$ indexes at $2 \mathrm{MAC}$ are higher than BIS ${ }^{\mathrm{TM}}$ indexes at $1 \mathrm{MAC}$, and as a consequence, mean values of BIS ${ }^{\mathrm{TM}}$ indexes do not increase monotonically with a decrease in DOA.

For a quantitative comparison between the inter-patient variability of BIS ${ }^{\mathrm{TM}}$ index and POM values, we normalized POM and $\mathrm{BIS}^{\mathrm{TM}}$ values of the patients by their respective dynamic ranges to equalize parameters scales and then derived their normalized standard deviations (NSD) as follows:

$$
\begin{aligned}
\operatorname{NSD}(t) & =\frac{\operatorname{SD}(t)}{\text { Dynamic Range }} \\
& =\frac{\sqrt{(1 / P-1) \sum_{p=1}^{P}\left(X_{p}(t)-\bar{X}_{p}(t)\right)^{2}}}{\left.\max \left(X_{p}(t)\right)\right|_{\forall_{t, p}}-\left.\min \left(X_{p}(t)\right)\right|_{\forall_{t, p}}}
\end{aligned}
$$

where $X$ may represent either CF08-POM, CF18-POM, CF28$\mathrm{POM}$ or BIS ${ }^{\mathrm{TM}} . p$ is the patient index which vary between 1 and $P=10$. The nominator represents the standard deviation of the parameter $\mathrm{X}$ at a given time, and the denominator expresses maximum range of that parameter in all patients during the twenty-minute EEG recordings.

Fig. 10(c) depicts NSD values of CF08-POM, CF18-POM, $\mathrm{CF} 28-\mathrm{POM}$ and BIS ${ }^{\mathrm{TM}}$ parameters in the five mentioned stages of anesthesia. NSD value of BIS ${ }^{\mathrm{TM}}$ parameter reaches its minimum and maximum values in stage 2 and stage 5 , respectively. The lowest NSD value in stages 4 and 5 (light anesthesia) is obtained by CF08-POM. CF28-POM has the lowest NSD values in stages 2 and 3 (moderate anesthesia), and finally in stage
1 (deep anesthesia) the lowest NSD value is rated to CF18POM.

\section{Discussion}

Analysis of SOM data shows that there are different delta sub-bands that may modulate alpha oscillations. Recognizing such distinguishable delta sub-bands is an indication that EEG delta waves may contain different activities induced by two or more different mechanisms in the brain. However, these mechanisms may not necessarily be independent from each other. Mechanisms underlying the production of these different delta sub-bands may work together or even have modulation effects on each other as they have on EEG oscillations in alpha band.

Dividing the delta band into different sub-bands by the SOM criterion seems to have some advantages over splitting the delta band by EEG power criterion. By SOM criterion, delta wave components are in fact separated according to their neurophysiologic roles. Here, this neurophysiologic function is the modulation of alpha waves. By the EEG power criterion, it is not easy to separate delta sub-bands and to distinguish their boundaries. We may even miss a sub-band where two (or more) EEG underlying mechanisms generate activities with overlapping frequency components. On the contrary, SOM can better distinguish these mechanisms. Basically, SOM is low for overlapping frequency components, and it is high for those frequencies that mainly belong to only one of the EEG underlying mechanisms. This increases the chance of separating delta sub-bands from each other. Indeed, interacting between different EEG underlying mechanisms causes generation of "noisy" MSs at overlapped frequencies. These frequencies indicate boundary frequencies of each underlying mechanism, and thus boundary frequencies of EEG sub-bands. SOM leads us to a better physiologically-based 
method for identifying delta sub-band boundaries. Based on this approach we could show that if DOA decreases, slow delta band moves a bit to higher frequencies (Fig. 7).

POM can be treated as a parameter that gives us some insights into status of information processing and time-coding in the brain. For example, F-POM can indicate the time-lag between the generation of a fast delta wave or clocklike delta oscillation $(\sim 2-3 \mathrm{~Hz})$ in the cortex and thalamus, respectively, and alpha activities that are generated in the thalamus [17,18,20,21]. This kind of time-coding information could not be extracted from the power spectrum of delta and alpha waves (the mostly used tool for determining DOA). It was shown that F-POM changes from a negative value at $2 \mathrm{MAC}$ to a positive value at $1 \mathrm{MAC}$. This rapid inversion of the phase might be an indication of the change of the location of mechanisms generating delta waves with DOA. Our hypothesis is that at $2 \mathrm{MAC}$, delta waves are mainly stereotyped clocklike rhythms originating in thalamic areas, and at $1 \mathrm{MAC}$ delta waves are mainly related to cortical areas. Indeed, from the literature we know that clocklike delta oscillations are generated when thalamus cells are in hyperpolarized state $[20,22]$. Possibly, in deep Desflurane anesthesia this condition can be fulfilled and thalamic cells can be hyperpolarized. As supporting arguments for this issue, firstly, it is known that anesthetic agents prolong the decay time of $\mathrm{GABA}_{\mathrm{A}}$ receptor, and have inhibitory effects on brain cells [23]; secondly, it has been shown that when anesthesia deepens, delta waves become more rhythmic [1,24], and thus they are more similar to clocklike rhythms that are originated in the thalamus.

Our findings in this study can be employed in the physiologically-based mean-field modeling of brain electrical activities in general anesthesia. Modulation of alpha band oscillations concurrently by very slow, slow and fast delta bands activities is a characteristic of the EEG that, to our knowledge, has not been considered yet in mean-field models designed for EEG signals in anesthesia [25-27]. However, recently the switching behavior of neural populations to UP and DOWN states in the delta frequency range, which may trigger the modulation of alpha oscillations, and its possible underlying mechanisms have been addressed without studying the relation between slow and fast components of EEG signals [28-30]. Mechanisms, which make relations between slow and fast EEG components, still need to be better characterized, and to be modeled mathematically in mean-field models.

Our study suggests that putting time-coding information of brain activities beside other more conventional parameters that are used for determining DOA such as BIS ${ }^{\mathrm{TM}}$ index, spectral edge frequency and median frequency [31] might leads us to improve the performance of determining DOA in some stages of anesthesia. However, this does not imply that time-coding information (here, POM) can be used alone for determining DOA because in some periods of times the desired time-coding information may be missed or interfered with other time-codings in the brain (especially, in transition states where the strength of time-codings become very low) and thus, results will not be trustable.

In this study, we considered different formations of slow delta sub-bands and fast delta sub-bands in order to find a delta sub- band that its corresponding POM has the best performance for determining DOA in different stages of anesthesia. It seems that there is no single delta sub-band with the best performance in different DOA. Results show that POMs relating to very slow sub-band are less correlated with DOA; POMs relating to slow delta sub-band are not good for determining DOA in deep anesthesia; and, POMs relating to fast delta sub-band do not have the best performance in light anesthesia but a reasonable performance. Consequently, these arguments imply that a fast delta sub-band (e.g. $\delta_{1.8}^{C F}$ ) is the best choice among different delta sub-bands that we analyzed. In addition, this study implicitly suggests that better methods should be found to combine the time-coding information relating to alpha and slow delta waves and the information, which exists in the relationship between alpha and fast delta waves.

\section{Conflicts of interest}

None.

\section{Acknowledgements}

Part of this work has been supported by the French Ministry of Foreign Affairs via the French Embassy in Tehran, Iran, and the center for international research and collaboration (ISMO), and by Egid-Gundishapur program.

\section{References}

[1] Constant I, Seeman R, et al. Sevoflurane and epileptiform EEG changes. Paediatr Anaesth 2005;15(4):266-74.

[2] Benoit O, Daurat A, et al. Slow $(0.7-2 \mathrm{~Hz})$ and fast $(2-4 \mathrm{~Hz})$ delta components are differently correlated to theta, alpha and beta frequency bands during NREM sleep. Clinical Neurophysiology 2000;111(12): 2103-6.

[3] Steriade M, Amzica F, et al. Synchronization of fast $(30-40 \mathrm{~Hz})$ spontaneous cortical rhythms during brain activation. J Neurosci 1996;16(1):392-417.

[4] Steriade M. Grouping of brain rhythms in corticothalamic systems. Neuroscience 2006;137(4):1087-106.

[5] Fell J, Elfadil H, et al. Human scalp recorded sigma activity is modulated by slow EEG oscillations during deep sleep. Int J Neurosci 2002;112(7):893-900.

[6] Molle M, Marshall L, et al. Grouping of spindle activity during slow oscillations in human non-rapid eye movement sleep. J Neurosci 2002;22(24):10941-7.

[7] Vanhatalo S, Palva JM, et al. Infraslow oscillations modulate excitability and interictal epileptic activity in the human cortex during sleep. Proc Natl Acad Sci U S A 2004;101(14):5053-7.

[8] Rampil IJ. A primer for EEG signal processing in anesthesia. Anesthesiology 1998;89(4):980-1002.

[9] Bruhn J, Bouillon TW, et al. Bispectral index (BIS) and burst suppression: revealing a part of the BIS algorithm. J Clin Monit Comput 2000;16(8):593-6

[10] Morimoto Y, Hagihira S, et al. The relationship between bispectral index and electroencephalographic parameters during isoflurane anesthesia. Anesth Analg 2004;98(5):1336-40 [table of contents].

[11] Sigl JC, Chamoun NG. An introduction to bispectral analysis for the electroencephalogram. J Clin Monit 1994;10(6):392-404.

[12] Mapleson WW. Effect of age on MAC in humans: a meta-analysis. Br J Anaesth 1996;76(2):179-85. 
[13] Tirel O, Wodey E, et al. The impact of age on bispectral index values and EEG bispectrum during anaesthesia with desflurane and halothane in children. Br J Anaesth 2006;96(4):480-5.

[14] Oppenheim AV, Schafer RW, et al. Discrete-Time Signal Processing. 2nd edition Prentice Hall; 1999.

[15] Proakis JG, Manolakis DK. Digital Signal Processing: Principles, algorithms and applications. 4th Edition. Prentice Hall; 2006.

[16] Orfanidis SJ. Introduction to Signal Processing. International Ed edition Prentice-Hal; 1995.

[17] Steriade M, Nunez A, et al. Intracellular analysis of relations between the slow $(<1 \mathrm{~Hz})$ neocortical oscillation and other sleep rhythms of the electroencephalogram. J Neurosci 1993;13(8):3266-83.

[18] Steriade M, Nunez A, et al. A novel slow $(<1 \mathrm{~Hz})$ oscillation of neocortical neurons in vivo: depolarizing and hyperpolarizing components. J Neurosci 1993;13(8):3252-65.

[19] Achermann P, Borbely AA. Low-frequency $(<1 \mathrm{~Hz})$ oscillations in the human sleep electroencephalogram. Neuroscience 1997;81(1):213-22.

[20] Steriade M, Dossi RC, et al. Network modulation of a slow intrinsic oscillation of cat thalamocortical neurons implicated in sleep delta waves: cortically induced synchronization and brainstem cholinergic suppression. J Neurosci 1991;11(10):3200-17.

[21] Timofeev I, Steriade M. Low-frequency rhythms in the thalamus of intactcortex and decorticated cats. J Neurophysiol 1996;76(6):4152-68.

[22] Steriade M, Contreras D, et al. The slow $(<1 \mathrm{~Hz})$ oscillation in reticular thalamic and thalamocortical neurons: scenario of sleep rhythm generation in interacting thalamic and neocortical networks. J Neurosci 1993;13(8):3284-99.
[23] Hentschke H, Schwarz C, et al. Neocortex is the major target of sedative concentrations of volatile anaesthetics: strong depression of firing rates and increase of GABAA receptor-mediated inhibition. Eur J Neurosci 2005;21(1):93-102.

[24] Molaee-Ardekani B, Shamsollahi MB, et al. An Investigation on Different EEG Patterns From Awake to Deep Anesthesia: Application to improve methods of determining depth of anesthesia. In: Kim SI, Suh TS, editors. World Congress on Medical Physics and Biomedical Engineering 2006, 14/1. New York: Springer Berlin Heidelberg; 2006. p. 909-12.

[25] Steyn-Ross ML, Steyn-Ross DA, et al. Modelling general anaesthesia as a first-order phase transition in the cortex. Prog Biophys Mol Biol 2004;85(2-3):369-85.

[26] Bojak I, Liley DT. Modeling the effects of anesthesia on the electroencephalogram. Phys Rev E Stat Nonlin Soft Matter Phys 2005;71(4 Pt 1):041902.

[27] Foster BL, Bojak I, et al. Population based models of cortical drug response: insights from anaesthesia. Cogn Neurodyn 2008;2(4):283-96.

[28] Molaee-Ardekani B, Senhadji L, et al. Brain activity modeling in general anesthesia: enhancing local mean-field models using a slow adaptive firing rate. Phys Rev E Stat Nonlin Soft Matter Phys 2007;76(4 Pt 1):041911.

[29] Wilson MT, Barry M, et al. Characteristics of temporal fluctuations in the hyperpolarized state of the cortical slow oscillation. Phys Rev E Stat Nonlin Soft Matter Phys 2008;77(6 Pt 1):061908.

[30] Hutt A, Longtin A. Effects of the anesthetic agent propofol on neural populations. Cogn Neurodyn 2009.

[31] Senhadji L, Wodey E, et al. Monitoring approaches in general anesthesia: a survey. Crit Rev Biomed Eng 2002;30(1-3):85-97. 